\title{
Innovative potential of enterprise from a differentiated approach standpoint based on the concept of innovations' diffusion
}

\author{
Vladimir Lazarev*, Anna Churashkina, and Tatiana Shulenkova
}

Ulyanovsk State Technical University, Ulyanovsk, Russia

\begin{abstract}
The article contains the key results of the study of the essence of the innovative potential of an enterprise with solving the problems of strategic management of modern enterprises operating in the context of the innovation of the economy. As a vector for improving the theoretical and methodological foundations for solving problems in the innovation sphere, a differentiated approach to understanding the essence of the innovative potential of an enterprise is proposed. The possibility and expediency of differentiation based on types (strategies) of innovative behavior, identified based on the concept of diffusion of innovations, have been substantiated. The article reflects the main provisions of the author's idea of the essence of the innovative potential of an enterprise within the framework of a given approach. The significance of the research results for solving theoretical, methodological, and applied problems is concretized.
\end{abstract}

\section{Introduction}

In modern economic realities, the external environment is characterized by a high rate of change and a high level of uncertainty. This circumstance leads to an increase in the role of innovative transformations in ensuring socioeconomic growth. Today there are conditions for the emergence of large-scale technical changes, forming a new technological base of economic systems. At the same time, researchers and analysts note that the importance of innovations in modern socio-economic development will continue to increase, and many traditional growth factors will demonstrate a downward trend in importance. These processes are viewed as a "long-term systemic challenge" facing the Russian economy [1].

The response is the transition to an innovative economic growth model as a priority of state policy, which is directly reflected in the focus of national projects, programs, and initiatives. In particular, 2021 has been declared the year of science and technology in Russia. This macroeconomic policy vector is implemented by creating an environment that encourages market participants to innovative activity. Therefore, this vector of macroeconomic policy predetermines the growth in the value of innovations to ensure the longterm competitiveness of Russian enterprises: over time, an increasing number of market entities will be interested in the implementation of innovative transformations, which will face increased competition on an innovative basis.
It should be emphasized that the dynamics of strengthening innovation activity are significant for the formation and support of the competitiveness of Russian enterprises. This process is consistent with global trends: "knowledge and innovation are becoming the most important factors in the long-term advantages of firms in the new economy" [2]. In addition, it is appropriate to note the importance of innovations not only for ensuring the leadership positions of enterprises but also for their "survival": an increase in the concentration of innovative activity initiates adaptation processes, which are also based on innovations. In other words, the presence of an innovative aspect in the activities of enterprises is gradually becoming imperative.

The shift in priorities of the competitive advantages formation of enterprises is associated with an adjustment of the focus of strategic management. One of the key tasks of strategic management is to ensure competitiveness in the long term. Thus, an extensive research area arises, associated with searching for ways to solve traditional problems based on innovation. A vast research area arises, associated with searching for ways to solve traditional problems based on innovation. This area is heterogeneous and represents a collection of overlapping subsets of research, one of which focuses on the formation, assessment, and implementation of innovative potential as the basis for the innovative development of enterprises. To solve the indicated applied problems, understanding the innovative potential of an enterprise as an economical category plays a critical role. The paper presents the results of studying the essence of the innovative potential of an enterprise from the perspective of strategic management.

\footnotetext{
$\bar{*}$ Corresponding author: v.lazarev@ulstu.ru
} 


\section{Approaches to considering the essence of the innovative potential of enterprises in economic science}

Analysis of specialized literature allows concluding that there is no universal approach to understanding the innovative potential of an enterprise. Traditionally, there are at least two coexisting approaches - the result-based approach and the content-based approach. The resulting approach focuses on reflecting the implementation of the innovative potential of enterprises by fixating the existing results of innovation. The meaningful approach is focused on disclosing the essence of innovation potential through the composition of the resources necessary for the implementation of innovative activities or through a set of components of a different nature (competencies, resources, mechanisms) that determine the ability to achieve innovative goals. Note that the approach that is focused on disclosing the essence of innovation potential through the composition of resources is a special case of a meaningful approach, which, due to its prevalence in classifications, is often singled out as an independent one [3, 4]. It is also appropriate to note the presence of attempts to link the effective and meaningful approaches, introducing the resulting component into the structure of the innovative potential of the enterprise. Their long-term coexistence indirectly confirms the viability of these approaches in science and practice.

This viability is explained by the multifaceted nature of the innovative potential as an economic category that predetermines many difficulties in the unification process. At the same time, there is no consensus of opinion even within the framework of a single approach. The lists of criteria for assessment vary, which reflects the variety of analysis methods. Also, the lists of components that require consideration vary. This fact signals already about the discrepancies in the essential understanding of the category under consideration. However, there is a general tendency towards universalization, despite the discrepancies in understanding the innovative potential essence of an enterprise, including its content. This trend is embodied in the fact that a significant number of attempts are being made to characterize a single structure of innovative potential, which can be used as the basis for selecting criteria for the subsequent quantitative and qualitative characteristics of its state. The potentials included in the composition act as structural components or a more complex arrangement can be used.

For example, in [5], it is proposed to consider the intellectual, marketing, organizational and managerial, financial, production, and technical and research potential. Moreover, in work [4], three blocks are distinguished in the structure of the innovative potential of enterprises - the resultant, resource, and management blocks. Each block is represented by several components, including organizational, financial, personnel, material, technical, and other components. After the presented concretization of the essence of innovative potential by referring to its structure, the transition to the system of indicators characterizing each component is carried out.

An integral indicator (index) is also calculated in some cases, reflecting the general state of the enterprise's innovative capabilities. The assessment results are the basis for making informed management decisions in terms of the formation and implementation of innovative potential, management of enterprise development based on innovations, and a wide range of related issues. Because of this fact, a significant part of the methods implies the interpretation of the results in the "high" / "low" categories (with varying degrees of detail).

The considered sequence schematically reflects the typical logic of actions when researching to improve the theoretical and methodological foundations for assessing the innovative potential of enterprises and/or solving issues that are naturally associated with this process. The above scheme allows tracing the close relationship between the tasks "defining the essence of the innovative potential of enterprises" - "assessing the innovative potential of enterprises" - "making management decisions in the designated area." In other words, it can be stated that the level of efficiency of solving a specific problem significantly affects the quality of solving problems that follow it in the chain. Therefore, it is advisable to look for the application of efforts for the comprehensive development of theoretical and methodological foundations both in the technology for solving each problem and in the fullest consideration of existing connections.

So, for example, in the development of assessment methods, researchers are guided by the construction of a system of criteria that will most fully characterize the readiness of an enterprise to solve problems based on innovation. At the same time, researchers pay attention to the issues of data availability for analysis, the simplicity of the calculations, the objectivity of the estimates obtained, the visibility of the presentation of the results, and many other criteria that seem to be the most significant to the developers. Assessment techniques are associated with tasks for the solution of which their results can be used. These methodologies are reflected in requirements such as the ability to conduct a comparative assessment and/or reflect separately the available potential and the level of its use. Practical examples are the introduction of integral indicators for individual components along with a general integral indicator of the innovative potential of enterprises. This technique allows obtaining detailed results, which is shown in [4].

The connection between applied problems and the concretization of the innovative potential of enterprises through the structure seems to be least used at the moment. The issue of forming a general idea of the structure of innovative potential is traditionally solved based on identifying groups of factors that affect the ability of an enterprise to carry out innovative activities and the level of success of this activity. At the same time, the main ways to improve this stage of the study mainly lie in taking into account advanced trends (such as globalization and digitalization). Trends are transforming the operating conditions of enterprises and 
the success factors in the innovation sphere and taking into account the specifics of industries. However, the result of this phase is a unified (unified) structure of innovation potential, according to which it is proposed to build a further assessment. In other words, the development of assessment tools demonstrates sufficient flexibility regarding the goals and objectives, the solution of which will be based on the results obtained. However, the solution to the issue of concretizing the structure of the innovative potential of enterprises is actually happening separately from the target settings. Namely, this structure affects methodological developments, while the number of tasks for which an assessment of the innovative potential of enterprises is required in the context of the economy's innovation is growing. These tasks are heterogeneous. There is a tendency to expand the boundaries of what is commonly referred to as innovation and innovation. This circumstance makes it possible to call into question the possibility of developing a unified idea of the structure of the innovative potential of enterprises, which makes it possible to solve all groups of problems most effectively.

Considering all of the above, it seems appropriate to consider the possibility and analyze the feasibility of implementing a differentiated approach to considering the essence of the innovative potential of an enterprise as a vector for improving the theoretical and methodological foundations for solving innovative problems. Due to the strategic orientation of the study, it is of interest to consider the variety of strategic alternatives available to enterprises. Therefore, the type (strategy) of innovative behavior is chosen as the basis for differentiation. There is no universally accepted classification of innovative strategies that unambiguously reflects the idea of alternatives in the area under study. Therefore, the specification of the list of types of innovative behavior was carried out based on the concept of diffusion of innovations.

\section{The process of diffusion of innovations as the basis for identifying types and strategies of innovative behavior}

The diffusion of innovation has many patterns, the totality of which allows the division of enterprises into several groups, depending on the form of participation in the designated process.

J. Schumpeter noted that the innovation, which was developed and applied by one enterprise, over time, "mastered" by others. Moreover, inclusion in the development process occurs at different speeds. The number of followers grows over time. This fact is explained by the gradual simplification of innovation implementation activities. The most challenging task is faced by the first in the chain; they are forced to overcome the most significant difficulties. At the same time, their success facilitates the development of innovation for the following processes: firstly, by the very demonstration of the possibility of achieving success, and secondly, by developing algorithms for solving part of the problems. In addition, some tasks lose relevance as the innovation spreads, simplifying the activities of followers. A similar trend applies to later groups of followers, which explains the gradual increase in their number [6]. The presented pattern reflects a key feature of the diffusion process, covering the period of time from the emergence of innovation to the moment when it ceases to be such, becoming a routine.

A sufficient basis for dividing enterprises into groups is the difference in the time of the entry of enterprises into the diffusion process and the connection between the moment of entry and the nature of the solved tasks. In other words, we can state that it is possible to classify types of innovative behavior based on the role of the enterprise in the diffusion process.

Let us turn to the expediency of such a division. It is pertinent to emphasize that the dynamics of a gradual decrease in the complexity of tasks solved by followers seems to be significant for the essential characteristics of each group of enterprises. These dynamics can serve as a basis for determining the factors and conditions that are necessary and sufficient for the implementation of a specific role.

There is interest in the relationship between diffusion and the benefits a company receives by participating in it. The prospect of innovative strategies lies in the fact that the initiation of innovation if accepted by the market, leads to the formation of competitive advantages. Competitive advantage tends to wane as innovation spreads. They completely lose their strength by the time innovation becomes routine. In turn, the need for market acceptance of innovation is a prerequisite for obtaining a competitive advantage on its basis.

The market selects viable innovations in respect of which the diffusion process is carried out in the future. This circumstance causes high risks for the "pioneers," and their decrease as innovation becomes routine. Both trends are capable of making a significant contribution to forming a comprehensive understanding of the types of innovative behavior identified based on the concept of diffusion. In addition, the use of the concept of diffusion of innovations as a theoretical basis for identifying types of innovative behavior has several more significant advantages:

- the concept of diffusion of innovations is sufficiently developed (there is a significant amount of research that reveals various aspects of this phenomenon); this fact determines the possibility of a comprehensive approach to identifying the characteristics of enterprises due to their role in diffusion, that is, we are talking about the possibility of ensuring a high level of objectivity of the results obtained based on the considered theoretical base;

- the mechanism of the diffusion process is characterized by a sufficiently high degree of uniformity for different types of innovations, which provides the breadth of coverage necessary for solving theoretical and applied problems, i.e., forms a relatively broad area of application of the results obtained.

The combination of the above characteristics makes it possible to state that the classification of types of innovative behavior based on the concept of diffusion 
of innovations is justified since it forms a theoretical basis that meets the goals and objectives of the research.

In the specialized educational and scientific literature $[7,8]$, it is customary to distinguish four groups of enterprises concerning their role in diffusion: innovators, early recipients, early majority, and lagging behind. The innovators are enterprises that act as a source of progress; their key area of competence is the generation of scientific and technical knowledge and the creation of innovations. Early adopters, also referred to as "pioneers," are focused on maximizing the speed with which an innovation can be adopted and brought to market. The enterprises of the early majority carry out the dissemination of the already mastered innovation based on the experience of the pioneer enterprises. Lagging enterprises are introducing "innovation" at a time when it has actually passed into the category of routine. It is important to understand that the name "lagging behind" characterizes the lag to mastering innovation, not the efficiency of the enterprise as a whole. The nature of introducing innovations into production provides the lagging enterprises with the possibility of functioning but not obtaining significant competitive advantages.

The theory of innovations defines all groups of subjects, except the first, as imitators. The reason is that the activity of enterprises does not ensure the emergence of innovation but only its diffusion. However, the most effective is a different type of innovative behavior from a strategic standpoint. Innovators and early adopters are focused on gaining a competitive advantage through the introduction and improvement of innovation. The early majority and lagging enterprises are characterized by an orientation towards operating in a new area, based on their existing competitive advantages of production, marketing, or other nature.

Thus, based on the concept of diffusion of innovation, a list of strategic alternatives has been formed, namely four types of innovative behavior that enterprises can implement.

\section{Features of the innovative potential of enterprises, considering their role in the diffusion of innovations}

The above classification of types of innovative behavior allows introducing differentiation in terms of the essential understanding of the innovative potential based on the requirements for it, set by the role of the enterprise in the diffusion process. In this context, the process of theoretical comprehension of the essence of the innovative potential of an enterprise takes the following form:

- identification of the specifics of the tasks solved by each of the four considered groups of enterprises in the diffusion process;

- determination of the characteristics of the enterprise, which determine the possibility of solving these problems and, as a consequence, the possibility of achieving an innovative goal (implementing a particular type of innovative behavior);
- establishing relationships and determining the degree of importance of the components of the innovative potential (identified at the previous stage) for the implementation of the chosen innovative path.

As a result of the implementation of a differentiated approach, the specificity of each type of innovative behavior is reflected in the composition and structure of the innovative potential of enterprises, determining their features.

At the first stage, an idea is formed about the structure of each enterprise's innovative potential. Let us characterize the basic goals and objectives of their activities and form the components of the first level of detail, in general terms, reflecting the company's readiness to implement the enlarged groups of tasks necessary to achieve the goal.

Objectively speaking, innovators are not focused on participation in the diffusion of innovation. Still, it results from their activities, which allows characterizing their role in diffusion as an initiator. This category of enterprises solves unique problems. The uniqueness of the tasks determines the impossibility of building activities based on the reproduction of existing samples due to their absence. Therefore, the first component in the enterprises' innovation potential should reflect the degree of readiness of the enterprise to generate ideas.

Accordingly, the second component in the structure of the innovative potential should cover the characteristics of the enterprise, which determine its readiness to implement the generated ideas. Pioneers, innovators need to solve all groups of problems to develop and implement innovation, having carried out a complete innovation cycle.

Key characteristics of early adopters distinguish them from other types of innovative behavior. Early recipients are focused on the diffusion, development, and improvement of innovation by implementing their innovation activities. They are also focused on gaining competitive advantages through the fastest possible inclusion in the diffusion process. Based on this, the most significant components of the innovative potential of this group of enterprises are:

- a block of characteristics that give an idea of the level of susceptibility to innovations. This group of enterprises must ensure both a high speed of obtaining information and the maximum coverage of the areas in which monitoring is carried out since unsatisfactory indicators for any of the indicated parameters actually exclude the possibility of playing this innovative role. Lag almost always results in a loss of competitive advantage.

- a block of characteristics reflecting the potential for improving innovation. For the diffusion phase in which early adopters operate, no consumer preference for innovation has yet been identified. This circumstance determines the high level of importance of improving and adapting innovation to consumers' needs to ensure competitive advantages.

The block of characteristics that reveals the readiness to spread the innovation seems to be less significant in the context of the enterprise profile. 
In contrast, early majority enterprises have a primary goal of spreading innovation, as the priority is to capture a significant share of a new market. The early majority enterprises use their competitive advantages, namely, access to resources, brand presence, developed distribution network, and other resources. This circumstance determines the weight of this component in the structure of innovation potential. At the same time, the very possibility of being included in the diffusion process is coupled with the ability to recognize innovations at the stage when diffusion will bring the maximum benefit.

Therefore, the enterprise must have a certain level of susceptibility. However, the requirements for the speed of reaction and the range of areas in which monitoring is necessary are significantly lower. The time factor as a whole is not so critical for this category of enterprises since, up to certain limits, the time lag can be partially compensated for by the superiority in the possibilities of solving the problems of the dissemination of innovation.

However, at the same time, an unsatisfactory reaction rate (below the threshold level for this category) will deprive the company of the opportunity to engage in diffusion in time, "throwing it" into the category of lagging behind. The level of involvement of the early majority enterprises in the tasks of improving innovation is limited to the implementation of improvements as needed. This process predetermines both the presence in the innovation potential of a component reflecting the readiness to implement this type of activity and a significantly lower level of its significance and requirements. Thus, it can be noted that the constituent elements of the innovative potential of the enterprises of the early majority coincide with the elements highlighted in the composition of the innovative potential of early recipients. However, the ratio of their significance and the requirements for them are fundamentally different.

The activity of the "lagging behind" is focused on covering mass demand and is characterized by low innovation activity. Therefore, the requirements for the level of innovation potential are minimal. In fact, it is enough for this group of enterprises to have the ability to overcome entry barriers and the possibilities of internal adaptation of activities for the implementation of "innovation."

Based on the above, we can conclude that the innovative potentials of innovators and imitators of different orders are different from each other. Each role requires a unique set of characteristics that an enterprise must possess, which is reflected in the unique structure of innovation potential.

Figure 1 schematically shows the structure of the innovation potential of enterprises for each type of innovative behavior in the first approximation and with the minimum possible detailing to obtain meaningful results. In the structure of the innovative potential, enlarged blocks of its characteristics (components) are highlighted, directly correlated with the key tasks, the effective solution of which is necessary for the successful implementation of each type of innovative behavior. The size of the segments reflects the degree of importance of each enlarged block of components for the possibility of achieving the goals and objectives set by the enterprise.
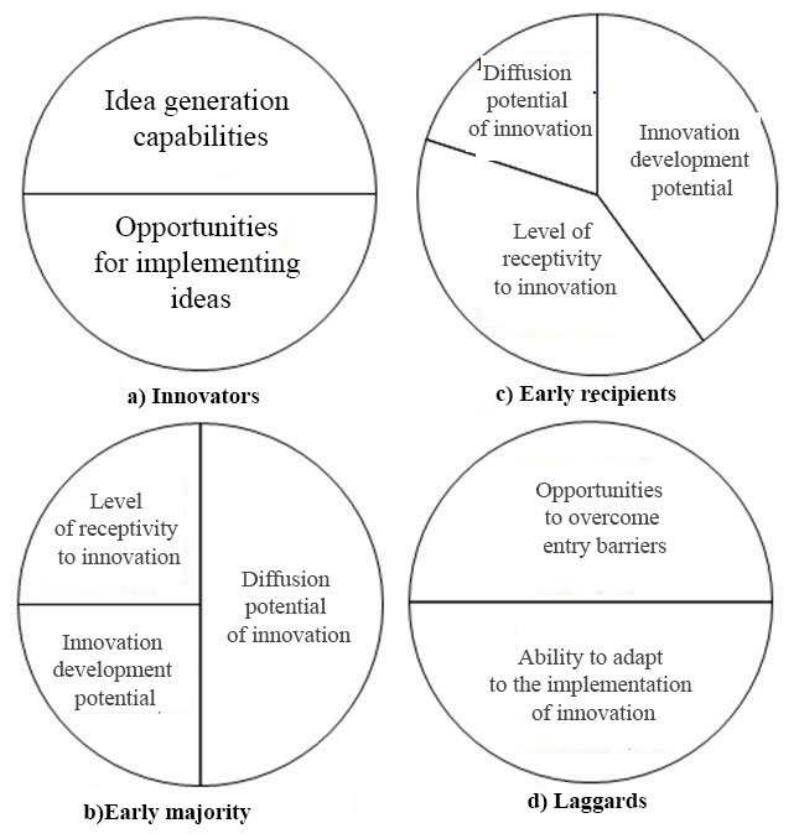

Figure 1. Visual representation of the features of the innovative potential of enterprises, depending on the implemented (planned for implementation) innovative role, compiled by the authors.

Despite the low level of detail, the model reflects a fundamental difference in the structure of capabilities that enterprises need to fulfill each of the roles, which predetermines the importance of further differentiation. This vision causes a shift in the focus of attention of researchers and decision-makers - from the search for a universal set of characteristics, the improvement of which lays the vector for increasing the innovative potential of the enterprise, to the analysis of combinations that form the growth potential within the framework of individual strategic roles. At this stage, it is also appropriate to focus on the fact that the solution of all designated groups of tasks is mandatory. This process leads to the requirement for a balanced structure as a determining criterion for the ability to play a particularly strategic role. In other words, if one or several components do not reach the minimum required level of development, then the potential as a whole cannot be considered satisfactory. Potential deficiencies are recognized even if other elements show superior development. This circumstance must be taken into account during the assessment, regardless of the methods used. The next level of detailing of the proposed model can be achieved by specifying the composition of each component while maintaining the logic of matching goals (subgoals) and enterprise characteristics that affect their attainability.

Thus, the component "the ability to generate ideas" can be concretized through the characteristics of the performance indicators of the $R \& D$ service, the assessment of the personnel composition of the enterprise and the level of openness inherent in the 
organizational culture, the analysis of the process of internal circulation of ideas and initiatives. Also, its composition may reflect the presence of its developments, patents (the resulting component). This fact demonstrates the flexibility of the model in terms of implementing basic approaches to understanding the essence of innovation potential. The relative simplicity of accounting for advanced concepts is also important to ensure the adequacy of the model to current conditions. Thus, what was listed earlier characterizes the emergence of ideas within the framework of the "closed innovation" paradigm, according to which this process is exclusively internal. It is possible to consider the "open innovation" paradigm, which allows the attraction of external sources of ideas - through the introduction of a component reflecting the level of interaction with external innovation structures.

At the same time, model varieties with different levels of detail are of practical importance. In particular, even with insignificant detailing of the model in the first approximation, it can be used for making managerial decisions in the role of a vector that sets the direction of analysis. This granularity is comparable to the level of detail of the Idea Generation component presented above. We use standard strategic management tools such as SNW analysis, focusing on decision-makers components and requirements. On this basis, it is possible to form a contour idea of the strengths and weaknesses of the enterprise and the gravitation of the innovative potential to the structure that best corresponds to a particular innovative role. This approach is entirely appropriate for express analysis for the initial diagnosis of the enterprise's innovative potential.

At the same time, the potential for further detailing the model is significant: an increase in the number of levels of analysis increases concreteness, providing the possibility of a gradual transition to a system of qualitative and quantitative indicators.

\section{Conclusions}

Summing up, let us concretize the advantages of a differentiated approach to considering the essence of innovation potential based on the concept of innovation diffusion:

- for theoretical developments: the proposed approach, due to the introduction of an appropriate target setting, allows at the initial stage of comprehension to take into account the multiplicity of strategic alternatives to innovative behavior, contributing to the convergence of theoretical concepts with the reality they represent, which has a positive effect on the applicability of the results obtained.

- for methodological developments: the proposed approach makes it possible to trace the motives for including specific components in the innovative potential by reflecting their influence on the possibility of implementing innovative strategies. In this regard, the results of estimates, the calculation of which will be based on the proposed theoretical model, will receive an additional layer of qualitative information essential for solving many applied problems.

- to improve the management process: the proposed approach contributes to the transformation of ideas about the process of formation and development of innovative potential, clearly demonstrating the limitations of the improvement strategy based solely on the characteristics of the level of development of individual indicators in isolation from the assessment of the structure of potential as a whole. So, going into innovators through improvement within the framework of the imitator's structure is not possible, and the development of individual characteristics of an innovator in the absence of a desire to fulfill this role and/or the absence of comprehensive development of the required structure seems unjustified.

Separately, it is appropriate to designate the traceable connection between the innovation diffusion process and the life cycle of industries (sub-sectors or areas of activity) [9], which suggests that there is a "request" for the implementation of a particular type of innovative behavior at a given moment in time. Because of this, other advantages can be indicated. A differentiated approach to studying the essence of the innovation potential of enterprises is possible, taking into account the concept of diffusion of innovation.

A differentiated approach deliberately ensures the comparability of the results of assessing external and internal factors when making management decisions regarding the implementation of innovative potential. A differentiated approach based on other features seems to be feasible. However, the composition of features and the feasibility of differentiation require additional research in each specific case.

\section{References}

1. The concept of long-term socio-economic development of the Russian Federation for the period up to 2020. Retrieved from: http://government.ru/info/6217/

2. V.S. Katkalo Evolution of the theory of strategic management (2011).

3. I.V. Antonenko Problems of the modern economy, 2, 33-37 (2010)

4. S.V. Terebova Scientific works: Institute for Economic Forecasting RAS, 15, 336-354 (2017)

5. O. I. Imaykina Izvestiya of higher educational institutions. Volga region. Social Sciences, 3, 211-223 (2014)

6. J.A Schumpeter Theory of Economic Development, (M.: Eksmo, 2008).

7. S. D. Ilyenkova Innovation Management (M: UNITY-DANA, 2012).

8. V.V. Manuilenko, A.A. Mishchenko Finance and Credit, 41, 21-31 (2012)

9. V.N. Lazarev, T.A. Shulenkova Organizational, economic and technological problems of modernizing the Russian economy: collection of articles of the VII International Scientific and Practical Conference, pp. 3-12 (2017). 